Chapter 11 in The Essential Guide to Image Processing, 2nd edition, pages:241-261.

Editor: Al Bovik, Academic Press, 2009

\title{
Multiscale Denoising of Photographic Images
}

\author{
Umesh Rajashekar and Eero P. Simoncelli \\ New York University
}

\section{Introduction}

Signal acquisition is a noisy business. In photographic images, there is noise within the light intensity signal (e.g., photon noise), and additional noise can arise within the sensor (e.g., thermal noise in a CMOS chip), as well as in subsequent processing (e.g., quantization). Image noise can be quite noticeable, as in images captured by inexpensive cameras built into cellular telephones, or imperceptible, as in images captured by professional digital cameras. Stated simply, the goal of image denoising is to recover the "true" signal (or its best approximation) from these noisy acquired observations. All such methods rely on understanding and exploiting the differences between the properties of signal and noise.

Formally, solutions to the denoising problem rely on three fundamental components: a signal model, a noise model, and finally a measure of signal fidelity (commonly known as the objective function) that is to be minimized. In this chapter, we'll describe the basics of image denoising, with an emphasis on signal properties. For noise modeling, we'll restrict ourselves to the case in which images are corrupted by additive, white, Gaussian noise - that is, we'll assume each pixel is contaminated by adding a sample drawn independently from a Gaussian probability distribution of fixed variance. A variety of other noise models and corruption processes are considered in Chapter 7. Throughout, we'll use the well known mean squared error (MSE) measure as an objective function.

We develop a sequence of three image denoising methods, motivating each one by observing a particular property of photographic images that emerges when they are decomposed into subbands at different spatial scales. We'll examine each of these properties quantitatively by examining statistics across a training set of photographic images and noise samples. And for each property, we'll use this quantitative characterization to develop two example denoising functions: a binary threshold function that retains or discards each multi-scale coefficient depending on whether it is more likely to be dominated by noise or signal, and a continuous-valued function that multiplies each coefficient by an optimized scalar value. Although these methods are quite simple, they capture many of the concepts that are used in state-of-the-art denoising systems. Toward the end of the chapter, we briefly describe several alternative approaches.

\section{Distinguishing Images from Noise in Multiscale Representations}

Consider the images in the top row of Figure 3. Your visual system is able to recognize effortlessly that the image in the left column is a photograph while the image in the middle column is filled with noise. How does it do this? We might hypothesize that it simply recognizes the difference in the distributions of pixel values in the two images. But the distribution of pixel values of photographic images is highly inconsistent from image to image, and more importantly, one can easily generate a noise image whose pixel distribution is matched to any given image (by simply spatially scrambling the pixels). So it seems that visual discrimination of photographs and noise cannot be accomplished based on the statistics of individual 


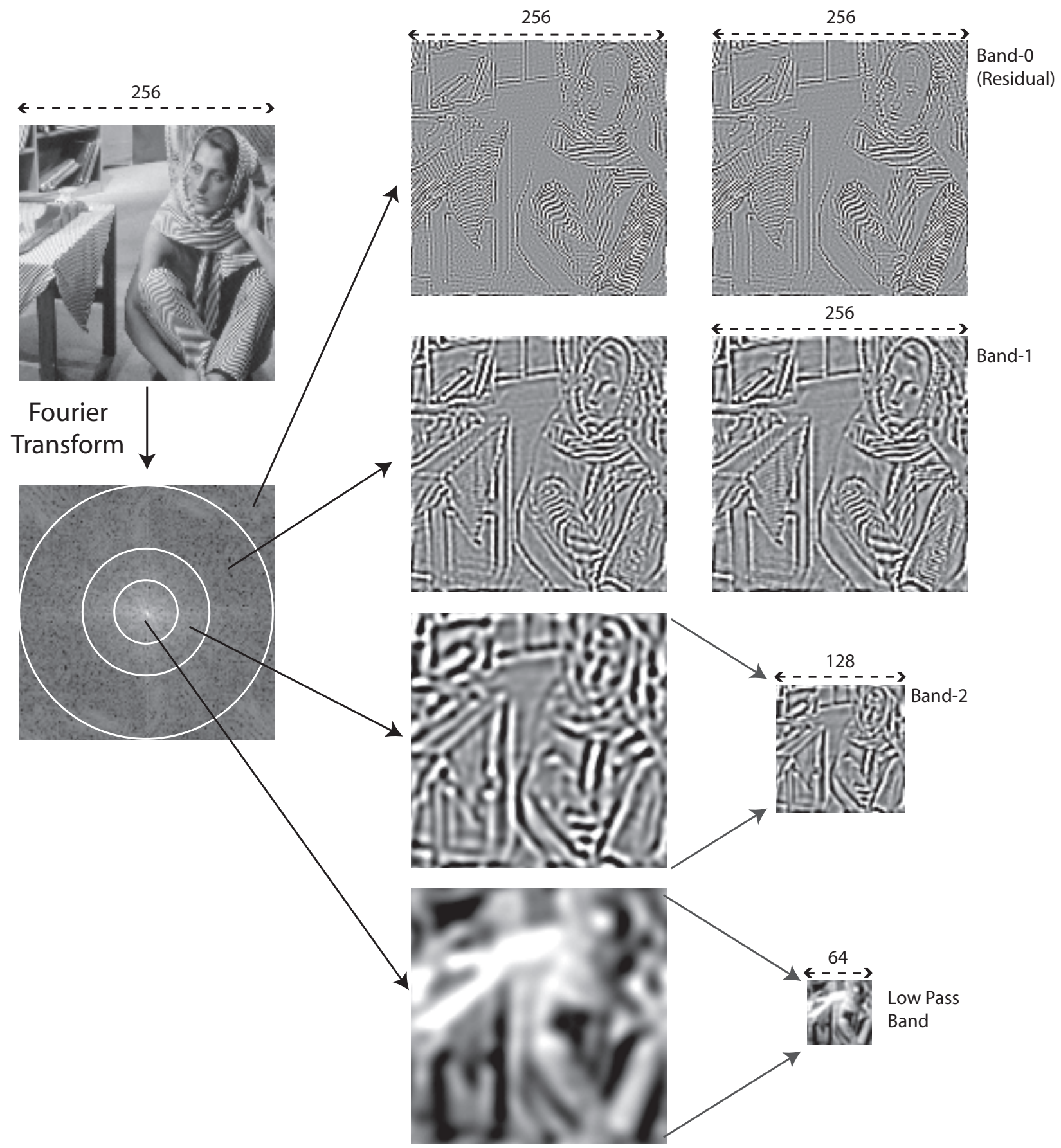

Figure 1: A graphical depiction of the multiscale image representation used for all examples in this chapter. Left column: An image and its centered Fourier transform. The white circles represent filters used to select bands of spatial frequencies. Middle column: Inverse Fourier transforms of the various spatial frequencies bands selected by the idealized filters in the left column. Each filtered image represents only a subset of the entire frequency space (indicated by the arrows originating from the left column). Depending on their maximum spatial frequency, some of these filtered images can be downsampled in the pixel domain without any loss of information. Right column: Downsampled versions of the filtered images in the middle column. The resulting images form the subbands of a multiscale "pyramid" representation $[1,2]$. The original image can be exactly recovered from these subbands by reversing the procedure used to construct the representation. 


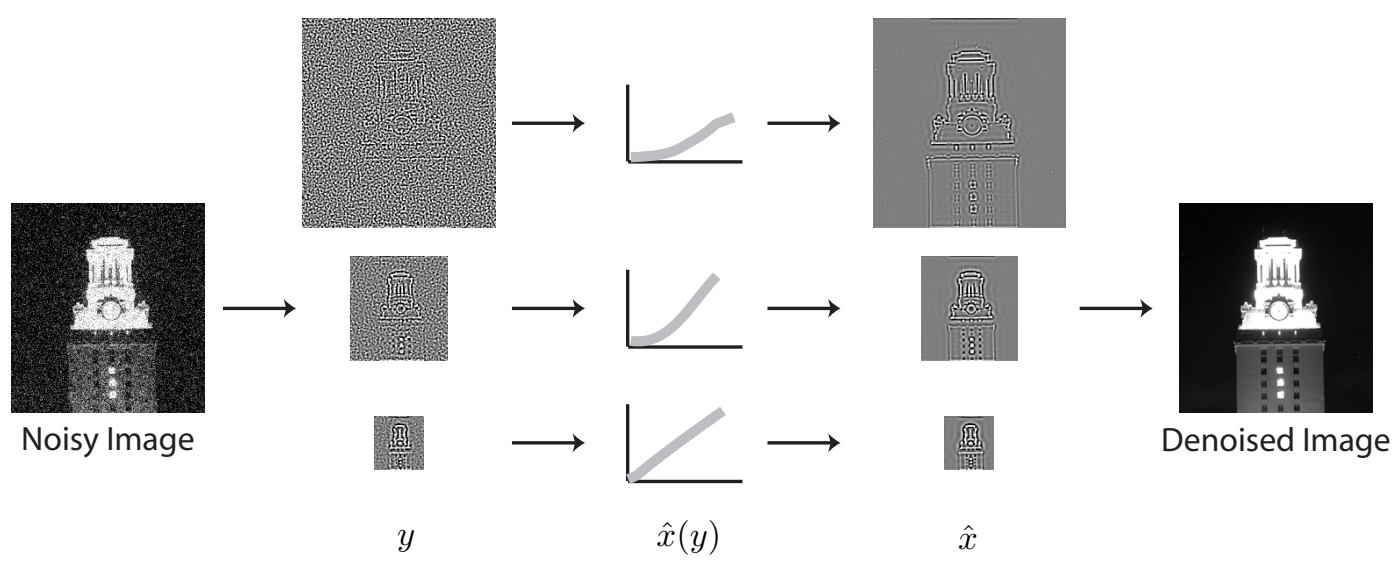

Figure 2: Block diagram of multiscale denoising. The noisy photographic image is first decomposed into a multiscale representation. The noisy pyramid coefficients, $y$, are then denoised using the functions, $\hat{x}(y)$, resulting in denoised coefficients, $\hat{x}$. Finally, the pyramid of denoised coefficients used to reconstruct the denoised image.

pixels. Nevertheless, the joint statistics of pixels reveal striking differences, and these may be exploited to distinguish photographs from noise, and also to restore an image that has been corrupted by noise, a process commonly referred to as denoising. Perhaps the most obvious (and historically, the oldest) observation is that spatially proximal pixels of photographs are correlated, whereas the noise pixels are not. Thus, a simple strategy for denoising an image is to separate it into smooth and non-smooth parts, or equivalently, low-frequency and a high-frequency components. This decomposition can then be applied recursively to the lowpass component to generate a multi-scale representation, as illustrated in Figure 1. The lower frequency subbands are smoother, and thus can be subsampled to allow a more efficient representation, generally known as a multiscale pyramid $[1,2]$. The resulting collection of frequency subbands contains the exact same information as the input image, but, as we shall see, it has been separated in such a way that it is more easily distinguished from noise. A detailed development of multiscale representations can be found in Chapter 6 of this book.

Transformation of an input image to a multiscale image representation has almost become a de facto preprocessing step for a wide variety of image processing and computer vision applications. For this chapter, we'll assume a three-step denoising methodology:

1. Compute the multiscale representation of the noisy image.

2. Denoise the noisy coefficients, $y$, of all bands except the low pass band using denoising functions $\hat{x}(y)$ to get an estimate, $\hat{x}$, of the true signal coefficient, $x$.

3. Invert the multiscale representation (i.e., recombine the subbands) to obtain a denoised image.

This sequence is illustrated in Figure 2. Given this general framework, our problem is to determine the form of the denoising functions, $\hat{x}(y)$.

\section{Subband Denoising - A Global Approach}

We begin by making some observations about the differences between photographic images and random noise. Figure 3 shows the multiscale decomposition of an essentially noise-free photograph, random noise, and a noisy image obtained by adding the two. The pixels of the signal (the noise-free photograph) lie in the interval $[0,255]$. The noise pixels are uncorrelated samples of a Gaussian distribution with zero mean and standard deviation of 60 . When we look at the subbands of the noisy image, we notice that band 1 of the 


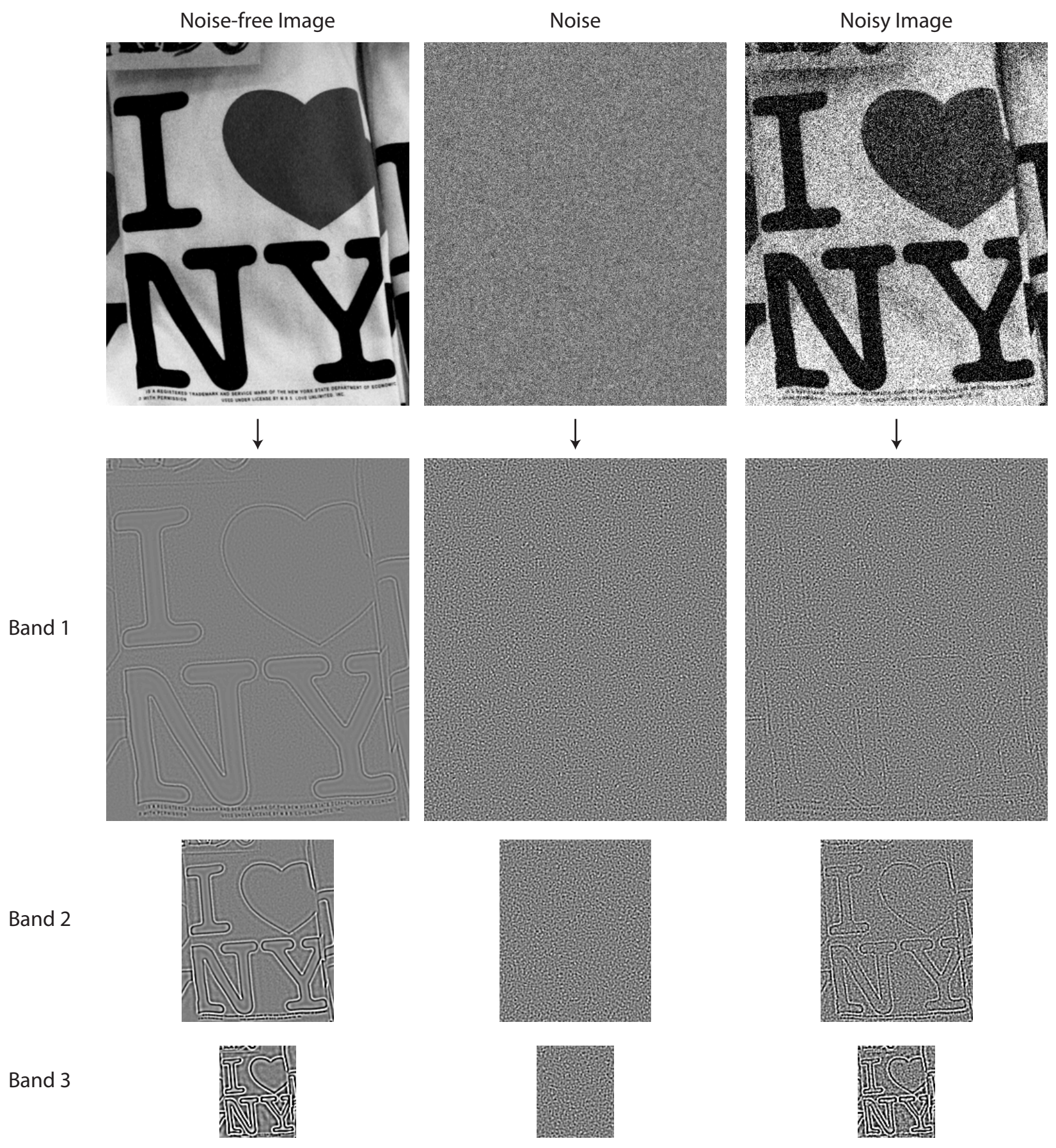

Figure 3: Multiscale representations of Left: of a noise-free photographic image. Middle: a Gaussian white noise image. Right: The noisy image obtained by adding the noise-free image and the white noise. 
(A)

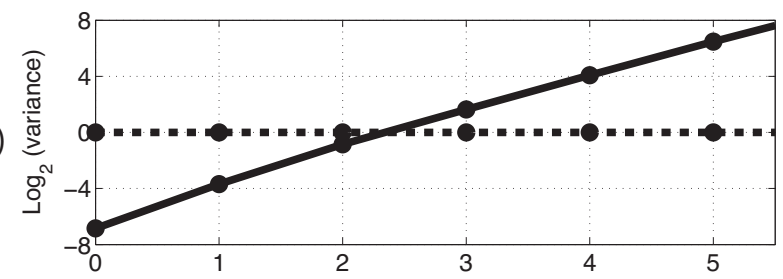

(B)

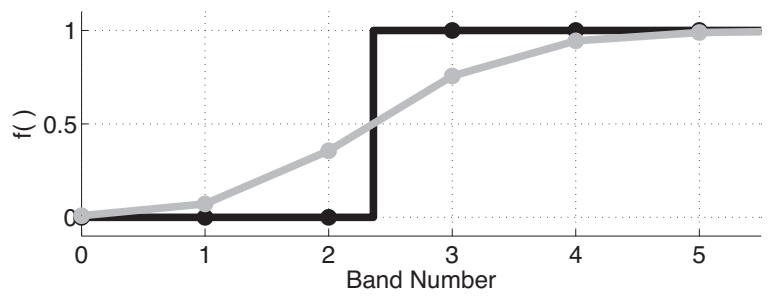

Figure 4: Band denoising functions. (A) Plot of average log variance of subbands of a multiscale pyramid as a function of the band number averaged over the photographic images in our training set (solid line, denoting $\log \left(|\vec{x}|^{2}\right)$ ) and Gaussian white noise image of standard deviation of 60 (dashed line, denoting $\log \left(|\vec{n}|^{2}\right)$. For visualization purposes, the curves have been normalized so that the log of the noise variance was equal to 0.0 (B) Optimal thresholding function (black) and weighting function (gray) as a function of band number.

noisy image is almost indistinguishable from the corresponding band for the noise image; band 2 of the noisy image is contaminated by noise, but some of the features from the original image remain visible; and band 3 looks nearly identical to the corresponding band of the original image. These observations suggest that, on average, noise coefficients tend to have larger amplitude than signal coefficients in the high frequency bands (e.g. band 1), whereas signal coefficients tend to be more dominant in the low frequency bands (e.g. band $3)$.

\subsection{Band Thresholding}

This observation about the relative strength of signal and noise in different frequency bands leads us to our first denoising technique: we can set each coefficient that lies in a band that is significantly corrupted by noise (e.g., band 1) to zero, and retain the other bands without modification. In other words, we make a binary decision to retain or discard each subband. But how do we decide which bands to keep and which to discard? To address this issue, let us denote the entire band of noise-free image coefficients as a vector, $\vec{x}$, the coefficients of the noise image as $\vec{n}$, and the band of noisy coefficients as $\vec{y}=\vec{x}+\vec{n}$. Then the total squared error incurred if we should decide to retain the noisy band is $|\vec{x}-\vec{y}|^{2}=|\vec{n}|^{2}$, and the error incurred if we discard the band is $|\vec{x}-\overrightarrow{0}|^{2}=|\vec{x}|^{2}$. Since our objective is to minimize the mean squared error between the original and denoised coefficients, the optimal decision is to retain the band whenever the signal energy (i.e., the squared norm of the signal vector, $\vec{x}$ ) is greater than that of the noise (i.e., $|\vec{x}|^{2}>|\vec{n}|^{2}$ ) and discard it otherwise ${ }^{1}$

To implement this algorithm, we need to know the energy (or variance) of the noise-free signal, $|\vec{x}|^{2}$, and noise, $|\vec{n}|^{2}$. There are several possible ways for us to obtain these.

- Method I: we can assume values for either or both, based on some prior knowledge or principles about images or our measurement device.

- Method II: we can estimate them in advance from a set of "training" or calibration measurements. For the noise, we might imagine measuring the variability in the pixel values for photographs of a set

\footnotetext{
${ }^{1}$ Minimizing the total energy is equivalent to minimizing the mean squared error, since the latter is obtained from the former by dividing by the number of elements.
} 
of known test images. For the photographic images, we could measure the variance of subbands of noise-free images. In both cases, we must assume that our training images have the same variance properties as the images that we will subsequently denoise.

- Method III: we can attempt to determine the variance of signal and/or noise from the observed noisy coefficients of the image we are trying to denoise. For example, if the noise energy is known to have a value of $E_{n}^{2}$, we could estimate the signal energy as $|\vec{x}|^{2}=|\vec{y}-\vec{n}|^{2} \approx|\vec{y}|^{2}-E_{n}^{2}$, where the approximation assumes that the noise is independent of the signal, and that the actual noise energy is close to the assumed value: $|\vec{n}|^{2} \approx E_{n}^{2}$.

These three methods of obtaining parameters may be combined, obtaining some parameters with one method and others with another.

For our purposes, we assume that the noise variance is known in advance (Method I), and we use Method II to obtain estimates of the signal variance by looking at values across a training set of images. Figure $4 \mathrm{~A}$ shows a plot of the variance as a function of the band number, for 30 photographic images $^{2}$ (solid line) compared with that of 30 equal-sized Gaussian white noise images (dashed line) of a fixed standard deviation of 60. For ease of comparison, we have plotted the logarithm of the band variance, and normalized the curves so that the variance of the noise bands is 1.0 (and hence the log variance is zero). The plot confirms our observation that, on average, noise dominates the higher frequency bands ( 0 through 2$)$ and signal dominates the lower frequency bands ( 3 and above). Furthermore, we see that the signal variance is nearly a straight line. Figure 4B shows the optimal binary denoising function (solid black line) that results from assuming these signal variances. This is a step function, with the step located at the point where the signal variance crosses the noise variance.

We can examine the behavior of this method visually, by retaining or discarding the subbands of the pyramid of noisy coefficients according to the optimal rule in Figure 4B, and then generating a denoised image by inverting the pyramid transformation. Figure $8 \mathrm{C}$ shows the result of applying this denoising technique to the noisy image shown in Fig. 8B. We can see that a substantial amount of the noise has been eliminated, although the denoised image appears somewhat blurred since the high frequency bands have been discarded. The performance of this denoising scheme can be quantified using the mean squared error (MSE), or with the related measure of peak signal-to-noise-ratio (PSNR), which is essentially a log-domain version of the mean squared error. If we define the MSE between two vectors $\vec{x}$ and $\vec{y}$, each of size $N$, as $M S E(\vec{x}, \vec{y})=\frac{1}{N}|\vec{x}-\vec{y}|^{2}$, then the PSNR (assuming 8-bit images) is defined as $\operatorname{PSNR}(\vec{x}, \vec{y})=10 \log _{10} \frac{255^{2}}{\operatorname{MSE}(\vec{x}, \vec{y})}$ and measured in units of decibels $(\mathrm{dB})$. For the current example, the PSNR of the noisy and denoised image were 13.40dB and $24.45 \mathrm{~dB}$ respectively. Figure 9 shows the improvement in PSNR over the noisy image across 5 different images.

\subsection{Band Weighting}

In the previous section, we developed a binary denoising function based on knowledge of the relative strength of signal and noise in each band. In general, we can write the solution for each individual coefficient:

$$
\hat{x}(\vec{y})=f(|\vec{y}|) \cdot y
$$

where the binary-valued function, $f(\cdot)$, is written as a function of the energy of the noisy coefficients, $|\vec{y}|$, to allow estimation of signal or noise variance from the observation (as described in Method III above). An examination of the pyramid decomposition of the noisy image in Figure 3 suggests that the binary assumption is overly restrictive. Band 1, for example, contains some residual signal that is visible despite the large amount of noise. And band 3 shows some noise in the presence of strong signal coefficients. This observation suggests that instead of the binary retain-or-discard technique, we might obtain better results by allowing $f(\cdot)$ to take on real values that depend on the relative strength of the signal and noise.

\footnotetext{
${ }^{2}$ All images in our training set are of New York City street scenes, each of size $1536 \times 1024$ pixels. The images were acquired using a Canon 30D digital SLR camera.
} 
But how do we determine the optimal real-valued denoising function $f(\cdot)$ ? For each band of noisy coefficients $\vec{y}$, we seek a scalar value, $a$, that minimizes the error $|a \vec{y}-\vec{x}|^{2}$. To find the optimal value, we can expand the error as $a^{2} \vec{y}^{T} \vec{y}-2 a \vec{y}^{T} \vec{x}+\vec{x}^{T} \vec{x}$, differentiate it with respect to $a$, set the result to zero, and solve for $a$. The optimal value is found to be

$$
\hat{a}=\frac{\vec{y}^{T} \vec{x}}{\vec{y}^{T} \vec{y}}
$$

Using the fact that the noise is uncorrelated with the signal (i.e. $\vec{x}^{T} \vec{n} \approx 0$ ) and the definition of the noisy image $\vec{y}=\vec{x}+\vec{n}$, we may express the optimal value as

$$
\hat{a}=\frac{|\vec{x}|^{2}}{|\vec{x}|^{2}+|\vec{n}|^{2}}
$$

That is, the optimal scalar multiplier is a value in the range $[0,1]$, which depends on the relative strength of signal and noise. As described under Method II in the previous section, we may estimate this quantity from training examples.

To compute this function $f(\cdot)$, we performed a five-band decomposition of the images and noise in our training set and computed the average values of $|\vec{x}|^{2}$ and $|\vec{n}|^{2}$, indicated by the solid and dashed lines in Figure 4A. The resulting function is plotted in gray as a function of the band number in Figure 4B. As expected, bands $0-1$, which are dominated by noise, have a weight close to zero; bands 4 and above, which have more signal energy, have a weight close to 1.0; and bands 2-3 are weighted by intermediate values. Since this denoising function includes the binary functions as a special case, the denoising performance cannot be any worse than band thresholding, and will in general be better. To denoise a noisy image, we compute its five-band decomposition, and weight each band in accordance to its weight indicated in Figure 4B and invert the pyramid to obtain the denoised image. An example of this denoising is shown in Figure 8D. The PSNR of the noisy and denoised images were $13.40 \mathrm{~dB}$ and $25.04 \mathrm{~dB}-$ an improvement of more than $11.5 \mathrm{~dB}$ ! This denoising performance is consistent across images, as shown in Figure 9.

Previously, the value of the optimal scalar was derived using Method II. But we can use the fact that $\vec{x}=\vec{y}-\vec{n}$, and the knowledge that noise is uncorrelated with the signal (i.e. $\vec{x}^{T} \vec{n} \approx 0$ ), to rewrite Eq. (2) as a function of each band as:

$$
\hat{a}=f(|\vec{y}|)=\frac{|\vec{y}|^{2}-|\vec{n}|^{2}}{|\vec{y}|^{2}} .
$$

If we assume that the noise energy is known, then this formulation is an example of Method III, and more generally, we now can rewrite $\hat{x}(\vec{y})=f(|\vec{y}|) \cdot y$.

The denoising function in Eq. (4) is often applied to coefficients in a Fourier transform representation, where it is known as the 'Wiener filter'. In this case, each Fourier transform coefficient is multiplied by a value that depends on the variances of the signal and noise at each spatial frequency- that is, the power spectra of the signal and noise. The power spectrum of natural images is commonly modeled using a power law, $F(\Omega)=A / \Omega^{p}$, where $\Omega$ is spatial frequency, $p$ is the exponent controlling the falloff of the signal power spectrum (typically near 2), $A$ is a scale factor controlling the overall signal power, is the unique form that is consistent with a process that is both translation- and scale-invariant (see Chapter 9). Note that this model is consistent with the measurements of Figure 4, since the frequency of the subbands grows exponentially with the band number. If, in addition, the noise spectrum is assumed to be flat (as it would be, for example, with Gaussian white noise), then the Wiener filter is simply

$$
|H(\Omega)|^{2}=\frac{\left|A / \Omega^{p}\right|}{\left|A / \Omega^{p}\right|+\sigma_{N}^{2}},
$$

where $\sigma_{N}^{2}$ is the noise variance. 


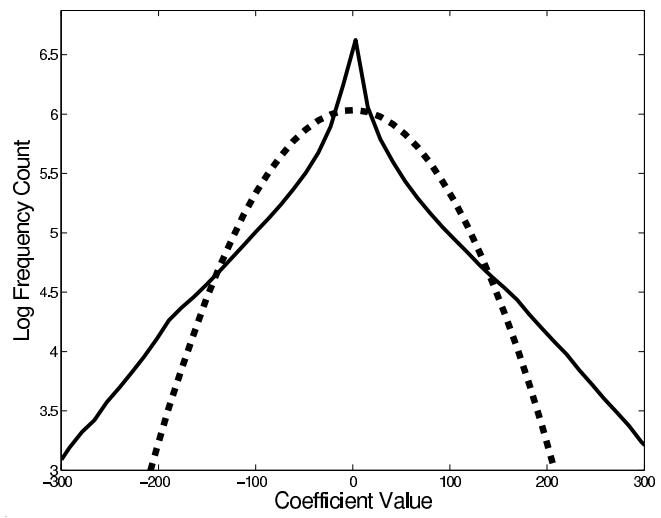

Figure 5: Log histograms of coefficients of a band in the multiscale pyramid for a photographic image (solid) and Gaussian white noise of standard deviation of 60 (dashed). As expected, the log of the distribution of the Gaussian noise is parabolic.

\section{Subband Coefficient Denoising - A Pointwise Approach}

The general form of denoising in the Section 3 involved weighting the entire band by a single number 0 or 1 for band thresholding, or a scalar between 0 and 1 for band weighting. However, we can observe that in a noisy band such as band 2 in Figure 3, the amplitudes of signal coefficients tend to be either very small, or quite substantial. The simple interpretation is that images have isolated features such as edges that tend to produce large coefficients in a multiscale representation. The noise, on the other hand, is relatively homogeneous.

To verify this observation, we used the 30 images in our training set and 30 Gaussian white noise images (standard deviation of 60) of the same size and computed the distribution of signal and noise coefficients in a band. Figure 5 shows the log of the distribution of the magnitude of signal (solid line) and noise coefficients (dashed line) in one band of the multiscale decomposition. We can see that the distribution tails are heavier and the frequency of small values is higher for the signal coefficients, in agreement with our observations above.

From this basic observation, we can see that signal and noise coefficients might be further distinguished based on their magnitudes. This idea has been used for decades in video cassette recorders for removing magnetic tape noise, where it is known as "coring". We capture it using a denoising function of the form:

$$
\hat{x}(y)=f(|y|) \cdot y
$$

where $\hat{x}(y)$ is the estimate of a single noisy coefficient $y$. Note that unlike the denoising scheme in Eq. (1), the value of the denoising function, $f(\cdot)$, will now be different for each coefficient.

\subsection{Coefficient-Thresholding}

Consider first the case where the function $f(\cdot)$ is constrained to be binary, analogous to our previous development of band thresholding. Given a band of noisy coefficients, our goal now is to determine a threshold such that coefficients whose magnitudes are less than this threshold are set to zero, and all coefficients whose magnitudes are greater than or equal to the threshold are retained.

The criterion for selecting the threshold is again selected so as to minimize the mean squared error. We determined this threshold empirically using our image training set. We computed the five-band pyramid for the noise-free and noisy images (corrupted by Gaussian noise of standard deviation of 60) to get pairs of noisy coefficients, $y$, and their corresponding noise-free coefficients, $x$, for a particular band. Let us now consider an arbitrary threshold value, say $T$. As in the case of band thresholding, there are two types of error introduced at any threshold level. First, when the magnitude of the observed coefficient, $y$, is below 

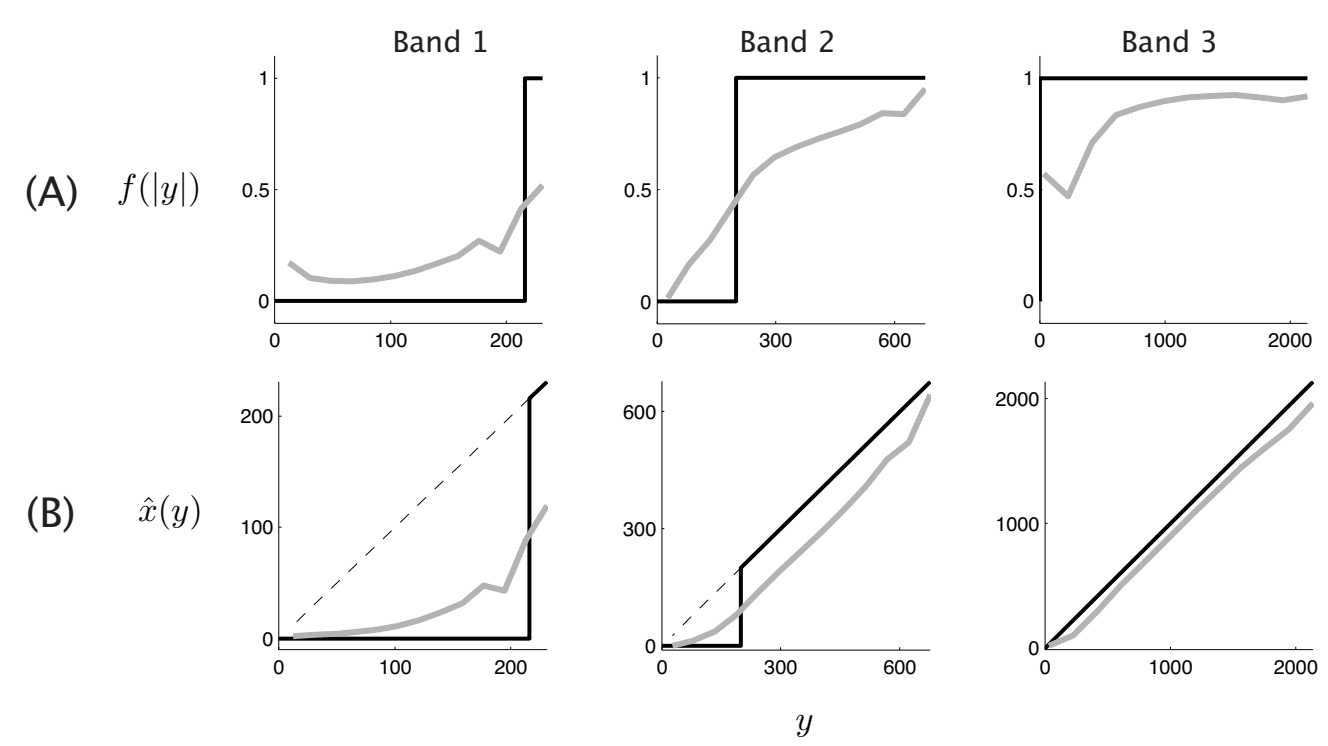

Figure 6: Coefficient denoising functions for three of the five pyramid bands. (A) Coefficient thresholding (black) and Coefficient weighting (gray) functions $f(|y|)$ as a function of $|y|$ (see Eq. (6)). (B) Coefficient estimation functions $\hat{x}(y)=f(|y|) \cdot y$. The dashed line depicts the unit slope line. For the sake of uniformity across the various denoising schemes, we show only one half of the denoising curve corresponding to the positive values of the observed noisy coefficient. Jaggedness in the curves occurs at values for which there was insufficient data to obtain a reliable estimate of the function.

the threshold and set to zero, we have discarded the signal, $x$, and hence incur an error of $x^{2}$. Second, when the observed coefficient is greater than the threshold, we leave the coefficient (signal and noise) unchanged. The error introduced by passing the noise component is $n^{2}=(y-x)^{2}$. Therefore, given pairs of coefficients, $\left(x_{i}, y_{i}\right)$, for a subband, the total error at a particular threshold, $T$, is

$$
\sum_{i:\left|y_{i}\right| \leq T} x_{i}^{2}+\sum_{i:\left|y_{i}\right|>T}\left(y_{i}-x_{i}\right)^{2}
$$

. Unlike the band denoising case, the optimal choice of threshold cannot be obtained in closed form. Using the pairs of coefficients obtained from the training set, we searched over the set of threshold values, $T$ to find the one that gave the smallest total least squared error.

Figure 6 shows the optimized threshold functions, $f(\cdot)$ in Eq. (6) as solid black lines for three of the five bands that we used in our analysis. For readers who might be more familiar with the input-output form, we also show the denoising functions $\hat{x}(y)$ in Figure 6B. The resulting plots are intuitive and can be explained as follows. For Band 1, we know that all the coefficients are likely to be corrupted heavily by noise. Therefore the threshold value is so high that essentially all of the coefficients are set to zero. For band 2, the signal-to-noise ratio increases and therefore the threshold values get smaller allowing more of the larger magnitude coefficients to pass unchanged. Finally, once we reach bands 3 and above, the signal is so strong compared to noise that the threshold is close to zero, thus allowing all coefficients to be passed without alteration.

To denoise a noisy image, we first decompose it using the multiscale pyramid, and apply an appropriate threshold operation to the coefficients of each band (as plotted in Figure 6). Coefficients whose magnitudes are smaller than the threshold are set to zero, and the rest are left unaltered. The signs of the observed coefficients are retained. Figure $8 \mathrm{E}$ shows the result of this denoising scheme, and additional examples of PSNR improvement are given in Figure 9. We can see that the coefficient-based thresholding has an improvement of roughly $1 \mathrm{~dB}$ over band thresholding. 
Although this denoising method is more powerful than the whole-band methods described in the previous section, note that it requires more knowledge of the signal and the noise. Specifically, the coefficient threshold values were derived based on knowledge of the distributions of both signal and noise coefficients. The former was obtained from training images, and thus relies on the additional assumption that the image to be denoised has a distribution that is the same as that seen in the training images. The latter was obtained by assuming the noise was white and Gaussian, of known variance. As with the band denoising methods, it is also possible to approximate the optimal denoising function directly from the noisy image data, although this procedure is significantly more complex than the one outlined above. Specifically, Donoho and Johnstone [3] proposed a methodology known as SUREshrink for selecting the threshold based on the observed noisy data, and showed it to be optimal for a variety of some classes of regular functions [4]. They also explored another denoising function, known as soft-thresholding, in which a fixed value is subtracted from the coefficients whose magnitudes are greater than the threshold. This function is continuous (as opposed to the hard thresholding function) and has been shown to produce more visually pleasing images.

\subsection{Coefficient-Weighting}

As in the band denoising case, a natural extension of the coefficient thresholding method is to allow the function $f(\cdot)$ to take on scalar values between 0.0 and 1.0. Given a noisy coefficient value, $y$, we are interested in finding the scalar value $f(|y|)=a$ that minimizies

$$
\sum_{i: y_{i}=y}\left(x_{i}-f\left(\left|y_{i}\right|\right) \cdot y_{i}\right)^{2}=\sum_{i: y_{i}=y}\left(x_{i}-a \cdot y\right)^{2} .
$$

We differentiate this equation with respect to $a$, set the result equal to zero, and solve for $a$ resulting in the optimal estimate $\hat{a}=f(|y|)=(1 / y) \cdot\left(\sum_{i} x_{i} / \sum_{i} 1\right)$. The best estimate, $\hat{x}(y)=f(|y|) \cdot y$, is therefore simply the conditional mean of all noisy coefficients, $x_{i}$, whose noisy coefficients are such that $y_{i}=y$. In practise, it is likely that no noisy coefficient has a value that is exactly equal to $y$. Therefore, we bin the coefficients such that $y-\delta \leq\left|y_{i}\right| \leq y+\delta$, where $\delta$ is a small positive value.

The plot of this function $f(|y|)$ as a function of $y$ is shown as a light gray line in Figure $6 \mathrm{~A}$ for three of the five bands that we used in our analysis; the functions for the other bands ( 4 and above) look identical to band 3 . We also show the denoising functions, $\hat{x}(y)$, in $6 \mathrm{~B}$. The reader will notice that, similar to the Band weighting functions, these functions are smooth approximations of the hard thresholding functions, whose thresholds always occur when the weighting estimator reaches a value of 0.5 .

To denoise a noisy image, we first decompose the image using a five-band multiscale pyramid. For a given band, we use the smooth function $f(\cdot)$ that was learned in the previous step (for that particular band), and multiply the magnitude of each noisy coefficient, $y$, by the corresponding value, $f(|y|)$. The sign of the observed coefficients are retained. The modified pyramid is then inverted to result in the denoised image as shown in Figure 8F. The method outperforms the coefficient thresholding method (since thresholding is again a special case of the scalar-valued denoising function). Improvements in PSNR over across five different images are shown in Figure 9.

As in the coefficient thresholding case, this method relies on a fair amount of knowledge about the signal and noise. Although the denoising function can be learned from training images (as was done here), this needs to be done for each band, and for each noise level, and it assumes that the image to be denoised has coefficient distributions similar to those of the training set. An alternative formulation, known as Bayesian coring was developed by Simoncelli and Adelson [5], who assumed a generalized Gaussian model (see Chapter 9) for the coefficient distributions. They then fit the parameters of this model adaptively to the noisy image, and then computed the optimal denoising function from the fitted model.

\section{Subband Neighborhood Denoising - Striking a Balance}

The technique presented in Section 3 was global, in that all coefficients in a band were multiplied by the same value. The technique in Section 4, on the other hand, was completely local: each coefficient was multiplied 
Band 1

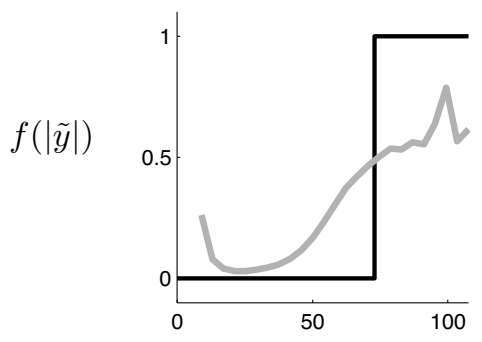

Band 2

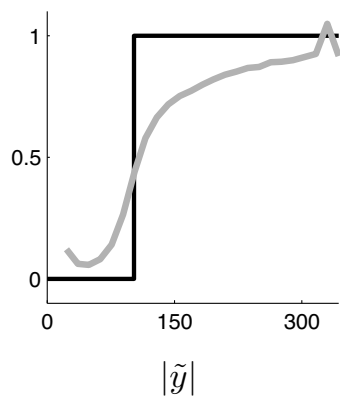

Band 3

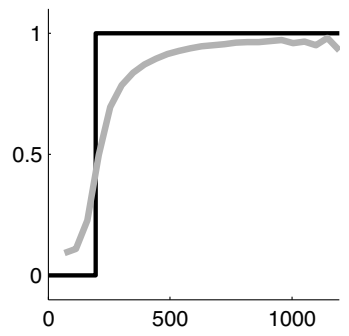

Figure 7: Neighborhood thresholding (black) and neighborhood weighting (gray) functions $f(|\tilde{y}|)$ as a function of $|\tilde{y}|$ (see Eq. (7) ) for various bands. Jaggedness in the curves occurs at values for which there was insufficient data to obtain a reliable estimate of the function.

by a value that depended only on the magnitude of that particular coefficient. Looking again at the bands of the noise-free signal in Figure 3, we can see that a method that treats each coefficient in isolation is not exploiting all of the available information about the signal. Specifically, the large magnitude coefficients tend to be spatially adjacent to other large magnitude coefficients (e.g., because they lie along contours or other spatially localized features). Hence, we should be able to improve the denoising of individual coefficients by incorporating knowledge of neighboring coefficients. In particular, we can use the energy of a small neighborhood around a given coefficient to provide some predictive information about the coefficient being denoised. In the form of our generic equation for denoising, we may write:

$$
\hat{x}(\tilde{y})=f(|\tilde{y}|) \cdot y
$$

where $\tilde{y}$ now corresponds to a neighborhood of multiscale coefficients around the coefficient to be denoised, $y$, and $|\cdot|$ indicates the vector magnitude.

\subsection{Neighborhood Thresholding}

Analogous to previous sections, we first consider a simple form of neighbor thresholding in which the function, $f(\cdot)$ in Eq. (7) is binary. Our methodology for determining the optimal function is identical to the technique previously discussed in Section 4.1, with the exception that we are now trying to find a threshold based on the local energy $|\tilde{y}|$ instead of the coefficient magnitude, $|y|$. For this simulation, we used a neighborhood of $5 \times 5$ coefficients surrounding the central coefficient.

To find the denoising functions, we begin by computing the five-band pyramid for the noise-free and noisy images in the training set. For a given subband we create triplets of noise-free coefficients, $x_{i}$, noisy coefficients, $y_{i}$, and the energy, $\left|\tilde{y}_{i}\right|$, of the $5 \times 5$ neighborhood around $y_{i}$. For a particular threshold value, $T$, the total error is given by

$$
\sum_{i:\left|\tilde{y}_{i}\right| \leq T} x_{i}^{2}+\sum_{i:\left|\tilde{y}_{i}\right|>T}\left(y_{i}-x_{i}\right)^{2}
$$


The threshold that provides the smallest error is then selected. A plot of the resulting functions, $f(\cdot)$ is shown by the solid black line in Figure 7 . The coefficient estimation functions, $\hat{x}(\tilde{y})$, depend on both $|\tilde{y}|$ and $y$ and not very easy to visualize. The reader should note that the abscissa is now the energy of the neighborhood, and not the amplitude of a coefficient (as in Figure 6A).

To denoise a noisy image, we first compute the five-band pyramid decomposition, and for a given band, we first compute the local variance of the noisy coefficient using a $5 \times 5$ window, and use this estimate along with the corresponding band thresholding function in Figure 7A to denoise the magnitude of the coefficient. The sign of the noisy coefficient is retained. The pyramid is inverted to obtain the denoised image. The result of denoising an noisy image using this framework is shown in Figure 8g.

The use of neighborhood (or "contextual") information has permeated many areas of image processing. In denoising, one of the first published methods was locally adapted version of the Weiner filter by Lee [6], in which the local variance in the pixel domain is used to estimate the signal strength, and thus the denoising function. This method is available in Matlab (through the function wiener2). More recently, Chang, Yu and Vetterli [7] used this idea in a spatially-adaptive thresholding scheme and derive a closed form expression for the threshold. A variation of this implementation known as NeighShrink [8] is similar to our implementation, but determines the threshold in closed form based on the observed noisy image, thus obviating the need for training.

\subsection{Neighborhood Weighting}

As in the previous examples, a natural extension of the idea of thresholding a coefficient based on its neighbors is to weight the coefficient by a scalar value that is computed from the neighborhood energy. Once again, our implementation to find these functions, is the similar to the one presented earlier for the coefficient-weighting in Section, 4.2. Given the triplets, $\left(x_{i}, y_{i},\left|\tilde{y}_{i}\right|\right)$, we now solve for the scalar, $f\left(\left|\tilde{y}_{i}\right|\right)$, that minimizes:

$$
\sum_{i:\left|\tilde{y}_{i}\right|=|\tilde{y}|}\left(x_{i}-f\left(\left|\tilde{y}_{i}\right|\right) \cdot y_{i}\right)^{2} .
$$

Using the same technique from earlier, the resulting scalar can be shown to be $f\left(\left|\tilde{y}_{i}\right|\right)=\sum_{i}\left(x_{i} y_{i}\right) / \sum_{i}\left(y_{i}{ }^{2}\right)$. The form of the function, $f(\cdot)$ is show in Figure 7.The coefficient estimation functions, $\hat{x}(\tilde{y})$, depend on both $|\tilde{y}|$ and $y$ and not very easy to visualize.

To denoise an image, we first compute its five-band multiscale decomposition. For a given band, we use a $5 \times 5$ kernel to estimate the local energy $|\vec{y}|$ around each coefficient $y$, and use the denoising functions in Figure 7 to multiply the central coefficient $y$ by $f(|\vec{y}|)$. The pyramid is then inverted to create the denoised image as shown in shown in Figure $8 \mathrm{~h}$. We see in Figure 9 that this method provides consistent PSNR improvement over other schemes.

The use of contextual neighborhoods is found in all of the highest performing recent methods. Miçhak et al. [9] exploited the observation that when the central coefficient is divided by the magnitude of its spatial neighbors, the distribution of the multiscale coefficients is approximately Gaussian (see also [10]) and used this to develop a Wiener-like estimate. Of course, the "neighbors" in this formulation need not be restricted to spatially adjacent pixels. Sendur and Selesnick [11] derive a bivariate shrinkage function, where the neighborhood $\tilde{y}$ contains the coefficient being denoised, and the coefficient in the same location at the next coarsest scale (the "parent"). The resulting denoising functions are a two-dimensional extension of those shown in Figure 6. Portilla et. al. [12] present a denoising scheme based on modeling a neighborhood of coefficients as arising from an infinite mixture of Gaussian distributions, known as a "Gaussian scale mixture". The resulting least-squares denoising function uses a more general combination over the neighbors than a simple sum of squares, and this flexibility leads to substantial improvements in denoising performance. The problem of contextual denoising remains an active area of research, with new methods appearing every month. 

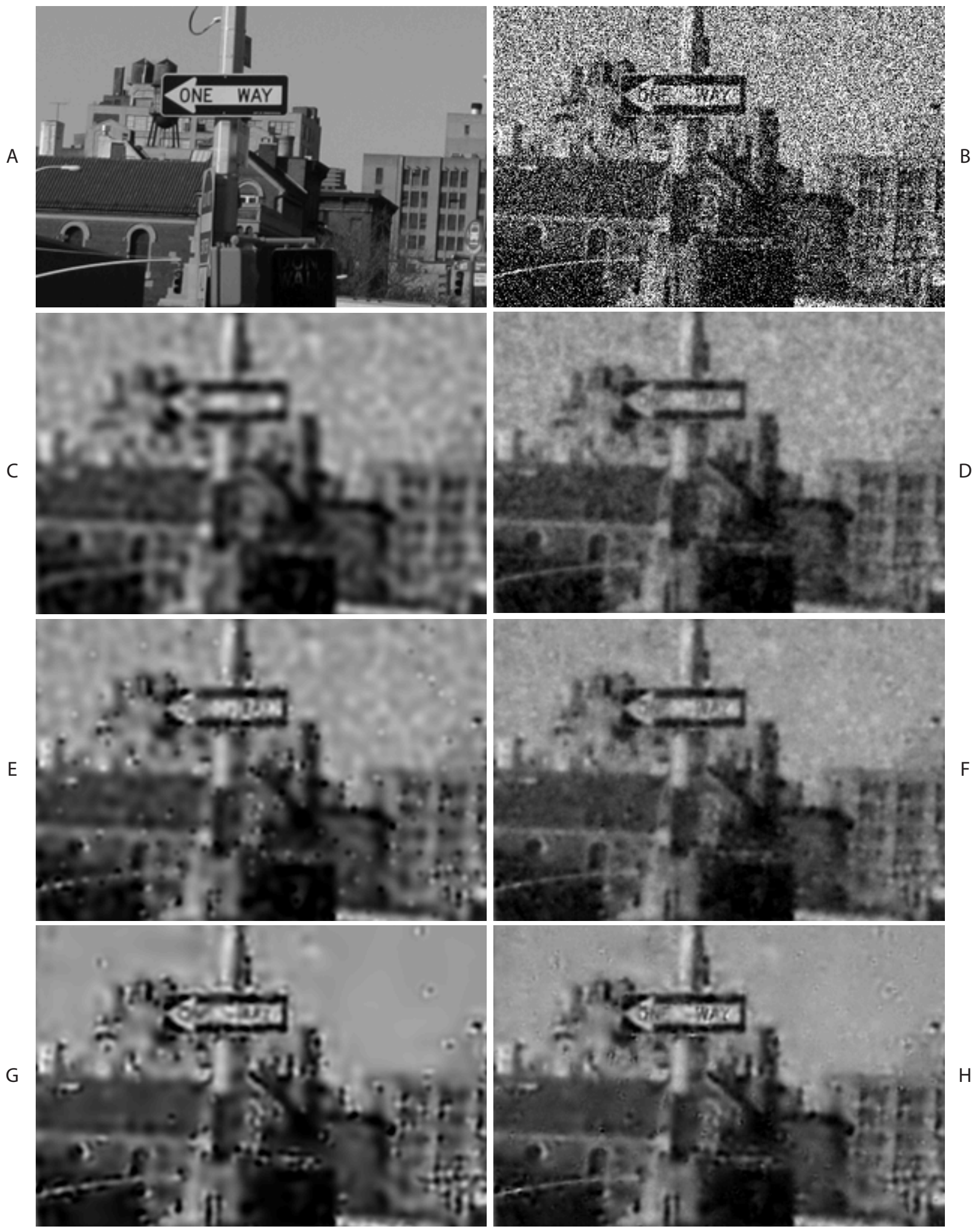

Figure 8: Example image Denoising results. (A) Original Image (B) Noisy Image (13.40dB) (C) Band Thresholding (24.45dB) (D) Band Weighting (25.04dB) (E) Coefficient Thresholding (24.97dB) (F) Coefficient Weighting (25.72dB) (G)Neighborhood Thresholding (26.24dB) $(\mathrm{H})$ Neighborhood Weighting (26.60dB). All images have been cropped from the original to highlight the details more clearly. 


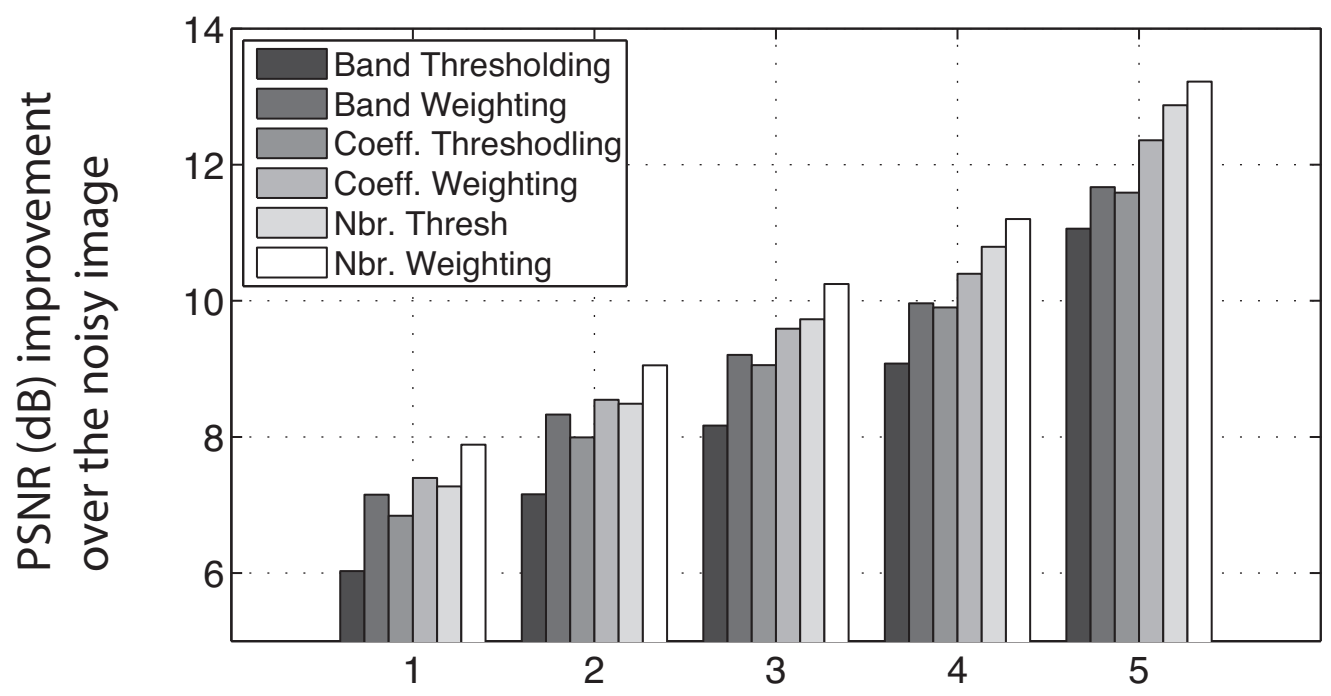

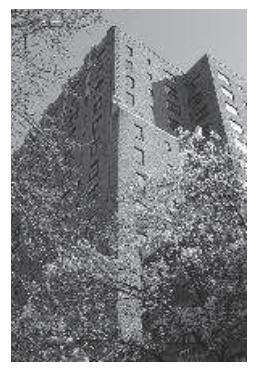

1

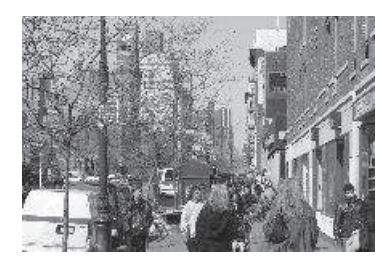

2

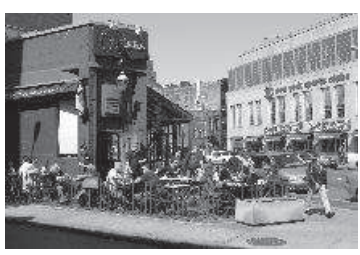

3

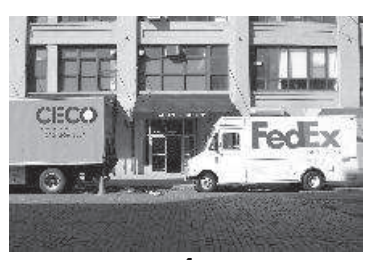

4

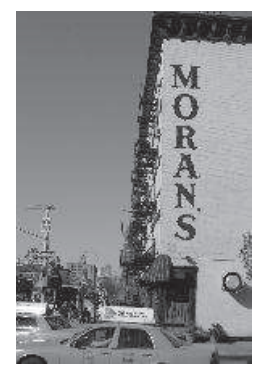

5

Figure 9: PSNR improvement (in $\mathrm{dB}$ ), relative to that of the noisy image. Each group of bars shows the performance of the six denoising schemes for one of the images shown in the bottom row. All denoising schemes used the exact same Gaussian white noise sample of standard deviation 60 .

\section{Statistical Modeling For Optimal Denoising}

In order to keep the presentation focused and simple, we have resorted to using a training set of noise-free and noisy coefficients to learn parameters for the denoising function (such as the threshold or weighting values). In particular, given training pairs of noise-free and noisy coefficients, $\left(x_{n}, y_{n}\right)$, we have solved a regression problem to obtain the parameters of the denoising function: $\hat{\theta}=\operatorname{argmin}_{\theta} \sum_{n}\left(x_{n}-f\left(y_{n} ; \theta\right) \cdot y_{n}\right)^{2}$. This methodology is appealing because it does not depend on models of image or noise, and this directness makes it easy to understand. It can also be useful for image enhancement in practical situations where it might be difficult to model the signal and noise. Recently, such an approach [13] was used to produce denoising results that are comparable to the state-of-the-art. As shown in that work, the data-driven approach can also be used to compensate for other distortions such as blurring.

But there are two clear drawbacks in the regression approach. First, the underlying assumption of such a training scheme is that the ensemble of training images is representative of all images. But some of the photographic image properties we've described, while general, do vary significantly from image to image, and it is thus preferable to adapt the denoising solution to the properties of the specific image being denoised. Second, the simplistic form of training we have described requires that the denoising functions must be separately learned for each noise level. Both of these drawbacks can be somewhat alleviated by considering a more abstract probabilistic formulation. 


\subsection{The Bayesian View}

If we consider the noise-free and noisy coefficients, $x$ and $y$, to be instances of two random variables $X$ and $Y$ respectively, we may rewrite the mean squared error criterion

$$
\begin{aligned}
\sum_{n}\left(x_{n}-g\left(y_{n}\right)\right)^{2} & \approx E_{X, Y}(X-g(Y))^{2} \\
& =\int \mathrm{d} X \int \mathrm{d} Y P(X, Y)(X-g(Y))^{2} \\
& =\int \mathrm{d} X \underbrace{P(X)}_{\text {Prior }} \int \mathrm{d} Y \underbrace{P(Y \mid X)}_{\text {Noise Model }} \underbrace{(X-g(Y))^{2}}_{\text {Loss function }}
\end{aligned}
$$

where $E_{X, Y}(\cdot)$ indicates the expected value, taken over random variables $X$ and $Y$.

As described earlier in Section 4.2, the denoising function, $g(Y)$, that minimizes this expression is the conditional expectation $E(X \mid Y)$. In the framework described above, we have replaced all our samples $\left(x_{n}, y_{n}\right)$ by their probability density functions. In general, the prior, $P(X)$, is the model for multiscale coefficients in the ensemble of noise-free images. The conditional density, $P(Y \mid X)$, is a model for the noise corruption process. Thus, this formulation cleanly separates the description of the noise from the description of the image properties, allowing us to learn the image model, $P(X)$, once and then re-use it for any level or type of noise since $(P(Y \mid X)$ need not be restricted to additive white Gaussian). The problem of image modeling is an active area of research, and is described in more detail in Chapter 9.

\subsection{Empirical Bayesian Methods}

The Bayesian approach, assumes that we know (or have learned from a training set) the densities $P(X)$ and $P(Y \mid X)$. While the idea of a single prior, $P(X)$, for all images in an ensemble is exciting and motivates much of the work in image modeling, denoising solutions based on this model are unable to adapt to the peculiarities of a particular image. The most successful recent image denoising techniques are based on empirical Bayes methods. The basic idea is define a parametric prior $P(X ; \theta)$ and adjust the parameters, $\theta$, for each image that is to be denoised. This adaptation can be difficult to achieve, since one generally has access only to the noisy data samples, $Y$, and not the noise-free samples, $X$. A conceptually simple method is to select the parameters that maximize the probability, but this utilizes a separate criterion (likelihood) for the parameter estimation and denoising, and can thus lead to suboptimal results. A more abstract but more consistent method relies on optimizing Stein's unbiased risk estimator (SURE) [14-17].

\section{Conclusions}

The main objective of this chapter was to lead the reader through a sequence of simple denoising techniques, illustrating how observed properties of noise and image structure can be formalized statistically and used to design and optimize denoising methods. We presented a unified framework for multiscale denoising of the form $\hat{x}(*)=f(*) \cdot y$, and developed three different versions, each one using a different definition for $*$. The first was a global model in which entire bands of multiscale coefficients were modified using a common denoising function, while the second was a local technique in which each individual coefficient was modified using a function that depended on its own value. The third approach adopted a compromise between these two extremes, using a function that depended on local neighborhood information to denoise each coefficient. For each of these denoising schemes, we presented two variations: a thresholding operator and a weighting operator.

An important aspect of our examples that we discussed only briefly is the choice of image representation. Our examples were based on an overcomplete multi-scale decomposition into octave-width frequency channels. While the development of orthogonal wavelets has had a profound impact on the application of compression, the artifacts that arise from the critical sampling of these decompositions are higly visible and 
detrimental when they are used for denoising. Since denoising generally less concerned about the economy of representation (and in particular, about the number of coefficients), it makes sense to relax the critical sampling requirement, sampling subbands at rates equal to or higher than their associated Nyquist limits. In fact, it has been demonstrated repeatedly (e.g., [18]) and recently proven [17] that redundancy in the image representation can lead directly to improved denoising performance. There has also been significant effort in developing multiscale geometric transforms such as Ridgelets, Curvelets, and Wedgelets which aim to provide better signal compaction by representing relevant image features such as edges and contours. And although this chapter has focused on multiscale image denoising, there have also been significant improvements in denoising in the pixel domain [19].

The three primary components of our the general statistical formalism of Eq. (8) - signal model, noise model, and error function - are all active areas of research. As mentioned previously, statistical modeling of images is discussed in Chapter 9. Regarding the noise, we've assumed an additive Gaussian model, but the noise that contaminates real images is often correlated, non-Gaussian, and even signal-dependent. Modeling of image noise is described in Chapter 7. And finally, there is room for improvement in the choice of objective function. Throughout this chapter, we minimized the error in the pyramid domain, but always reported the PSNR results in the image domain. If the multiscale pyramid is orthonormal, minimizing error in the multiscale domain is equivalent to minimizing error in the pixel domain. But in over-complete representations, this is no longer true, and noise that starts out white in the pixel domain is correlated in the pyramid domain. Recent approaches in image denoising attempt to minimize the mean-squared error in the image domain while still operating in an over-complete transform domain [13,17]. But even if the denoising scheme is designed to minimize PSNR in the pixel domain, it is well known that PSNR does not provide a good description of perceptual image quality (see Chapter 20). An important topic of future research is thus to optimize denoising functions using a perceptual metric for image quality [20].

\section{Acknowledgments}

All photographs used for training and testing were taken by Nicolas Bonnier. We thank Martin Raphan and Siwei Lyu for their comments and suggestions on the presentation of the material in this chapter.

\section{References}

[1] E. H. Adelson, C. H. Anderson, J. R. Bergen, P. J. Burt, and J. M. Ogden, "Pyramid methods in image processing," RCA Engineer, vol. 29, no. 6, pp. 33-41, 1984.

[2] P. J. Burt and E. H. Adelson, "The laplacian pyramid as a compact image code," IEEE Transactions on Communications, vol. COM-31, pp. 532-540, Apr. 1983.

[3] D. L. Donoho and I. M. Johnstone, "Ideal spatial adaptation by wavelet shrinkage," Biometrika, vol. 81, no. 3, pp. 425-455, 1994.

[4] D. L. Donoho and I. M. Johnstone, "Adapting to unknown smoothness via wavelet shrinkage," Journal of the American Statistical Association, vol. 90, no. 432, pp. 1200-1224, 1995.

[5] E. P. Simoncelli and E. H. Adelson, "Noise removal via bayesian wavelet coring," in Image Processing, 1996. Proceedings., International Conference on (E. Adelson, ed.), vol. 1, pp. 379-382 vol.1, 1996.

[6] J. S. Lee, "Digital image enhancement and noise filtering by use of local statistics," vol. PAMI-2, pp. 165-168, March 1980.

[7] S. G. Chang, B. Yu, and M. Vetterli, "Spatially adaptive wavelet thresholding with context modeling for image denoising," Image Processing, IEEE Transactions on, vol. 9, no. 9, pp. 1522-1531, 2000. 
[8] G. Chen, T. Bui, and A. Krzyzak, "Image denoising using neighbouring wavelet coefficients," in Acoustics, Speech, and Signal Processing, 2004. Proceedings. (ICASSP '04). IEEE International Conference on (T. Bui, ed.), vol. 2, pp. ii-917-20 vol.2, 2004.

[9] M. Kivanç Mihçak, I. Kozintsev, K. Ramchandran, and P. Moulin, "Low-complexity image denoising based on statistical modeling of wavelet coefficients," Signal Processing Letters, IEEE, vol. 6, no. 12, pp. 300-303, 1999.

[10] D. L. Ruderman and W. Bialek, "Statistics of natural images: Scaling in the woods," Phys. Rev. Letters, vol. 73, no. 6, pp. 814-817, 1994.

[11] L. Sendur and I. W. Selesnick, "Bivariate shrinkage functions for wavelet-based denoising exploiting interscale dependency," Signal Processing, IEEE Transactions on, vol. 50, no. 11, pp. 2744-2756, 2002.

[12] J. Portilla, V. Strela, M. Wainwright, and E. Simoncelli, "Image denoising using scale mixtures of gaussians in the wavelet domain," Image Processing, IEEE Transactions on, vol. 12, no. 11, pp. 13381351, 2003.

[13] Y. Hel-Or and D. Shaked, "A discriminative approach for wavelet denoising," Image Processing, IEEE Transactions on, vol. 17, no. 4, pp. 443-457, 2008.

[14] D. L. Donoho, "Denoising by soft-thresholding," IEEE Trans. Info. Theory, vol. 43, pp. 613-627, 1995.

[15] J. C. Pesquet and D. Leporini, "A new wavelet estimator for image denoising," in 6th International Conference on Image Processing and its Applications, (Dublin, Ireland), pp. 249-253, July 1997.

[16] F. Luisier, T. Blu, and M. Unser, "A new SURE approach to image denoising: Interscale orthonormal wavelet thresholding," IEEE Trans. Image Proc., vol. 16, March 2007.

[17] M. Raphan and E. P. Simoncelli, "Optimal denoising in redundant bases," IEEE Trans Image Processing, vol. 17, pp. 1342-1352, aug 2008.

[18] R. R. Coifman and D. L. Donoho, "Translation-invariant de-noising," in Wavelets and statistics (A. Antoniadis and G. Oppenheim, eds.), San Diego: Springer-Verlag lecture notes, 1995.

[19] K. Dabov, A. Foi, V. Katkovnik, and K. Egiazarian, "Image denoising by sparse 3-d transform-domain collaborative filtering," Image Processing, IEEE Transactions on, vol. 16, no. 8, pp. 2080-2095, 2007.

[20] S. S. Channappayya, A. C. Bovik, C. Caramanis, and R. W. Heath, "Design of linear equalizers optimized for the structural similarity index," Image Processing, IEEE Transactions on, vol. 17, pp. 857-872, June 2008. 